\title{
Research Motion Blurred Image Restoration Algorithms
}

\author{
Qiong Li, Junhui Rao and Xiaofeng Yang \\ College of Electronics \& Communication Engineering, \\ YuLin Normal University, YuLin, Guangxi 537000, China \\ yllq2000@163.com
}

\begin{abstract}
Subject in the process of image acquisition and the relative games between the camera or camera image fuzzy, it seriously affected the further image feature extraction, target recognition and image analysis. In order to meet the needs of reality, get high quality image, we must be used image restoration techniques for fuzzy image restoration. Based on the detailed study on the basis of the theory of wavelet transform, we confirmed by experiments in motion blurred image of wavelet and decomposition level .The method to identify the fuzzy parameters, under the condition of without noise and with noise on the four classic image restoration algorithm, the simulation experiment using the peak signal-to-noise ratio, mean square error, average gray level gradient method and Laplace operator method of four kinds of image quality evaluation index to evaluate the healing effect and recovery result diagram and its quality evaluation. Through the scene shooting motion blurred image point spread function of parameter identification and image restoration, it demonstrates that the proposed point spread function's parameters identification method is sound and effective.
\end{abstract}

Keywords: Motion Blurred, Two-dimensional Discrete Wavelet Transform, Cepstrum, Image Restoration

\section{Introduction}

The present era is the era of information overflowing, according to statistics, it about $80 \%$ above a person to get information from the visual that is obtained from the image. In daily life, we often contact images, such as photographs, pictures, books, video and so on, so the image and human life are inseparable. As the saying goes "stick out a mile", "seeing is believing" all that humans engage in all activities of the image is an important information source.

Due to the rapid development of modern digital technology, using digital image as digital information carrier has been the people's attention [1]. At present, the digital image has been widely used in various fields, such as medical, military and transportation etc.. But these applications require a higher quality of digital image, in order to meet the human needs of image observation or computer analysis of the requirement of image. The so-called image of high quality is to get clear image, it will not produces distortion, namely the image was not degenerate. However, the image acquisition requires a certain process, in the process of capturing imag es more or less will be affected by various factors, resulting in the quality of the image to produce degradation.

The most typical causes image degradation has the following five points:

(1) Receiving solar radiation, atmospheric turbulence and clouds obscured other factors, which makes the image distortion when imaging.

* Foundation item: Supported by the Science and Technology Projects of Guangxi Universities and Colleges(2013YB192), youth fundation of Yulin normal university (2010YJQN31), youth fundation of yulin normal university (2012YJQN25) 
(2) Relative movement between the imaging device and the target object produces motion blur.

(3) Focus at different depths exist or do not allow to target imaging region will produce defocus blur.

(4) The digital image forming system, the aberration, nonlinear distortion and limited bandwidth and other defects that the imaging device own exists which led to distortion of the image

(5) By working devices, optical and roads all kinds of noise introduced by digital image imaging systems.

Image degradation seriously affected the further image feature extraction, object recognition and image analysis. If we do not remedy, it will not only affect the use value of the image, and will make some key image without practical value. In order to meet the real needs, and get high-quality images ${ }^{[2]}$, we need to use better equipment or improve imaging environment. However, in many cases, it need the high cost of inputs and even inoperable, therefore, with the development of digital image processing technology, image restoration techniques has become a key information indispensable method that in order to maximize the recovery of degraded images .

In recent years, image restoration is an important branch in the field of digital image processing, and it has increasingly become the focus of attention [3-4]. Image restoration is the use of a priori knowledge of the degradation of the image to rebuild or restore the degraded image. It is mainly through a pre-determined target to improve the image, it can improve the visual effect of the image, but also in order to facilitate subsequent processing.

In the process of acquiring image, as mentioned above cause the image to produce degradation of many reasons. Image motion blur is that during filming, photographed occurred relative motion between the scene and imaging equipment, which obtained fuzzy image production. In reality, motion blur is prevalent, such as in the field of aerospace, high-speed flying spacecraft captured images of the target object are captured with motion blur, which hinders the human explore space; in aircraft remote sensing and satellite remote sensing technology, the investigation of aircraft or satellite captured a large number of aerial photographs are blurred, which is not conducive to extract useful intelligence; in traffic safety, the electronic eye that traffic junctions installed capturing high-speed car, the illegal vehicles captured images are blurred, which seriously affect traffic safety supervision departments and law enforcement. In addition, in real life, whether it is a hundred thousand type of professional camera, or a wide variety of digital cameras and mobile phones with powerful camera features, if the target object have motion during the shooting, and the resulting photos have motion blur, which greatly reduces the image quality, it does not meet expectations.

In the 1960s, space exploration have begun to study the recovery of the motion blurred image, such as 1964, California 's Jet Propulsion Laboratory [5], using gray-scale transformation, de-noising and other methods, it used the space probe to send back moon photo as experimental subjects, finally, it succeeded in drawing out the surface images of the lunar. Then, using the "Prowler 7th " accumulated image processing method, "Explorer" moon flight, " Mariner " Mars overflight mission, and "Apollo" human lunar exploration and other tasks sent back photos, using these photos made deconvolution, moved the motion blur and other image restoration processing, in order to improve the image quality significantly.

From the 1960s to now, motion blur image restoration has been developing in the land of vitality. In recent decades, the scope of application of the motion blur image restoration technique has been extended to a large number of scientific and technical fields, such as various aspects of the aerospace, military reconnaissance, road traffic 
monitoring, health care and so on. Therefore, it has important practical significance to study motion blur image restoration technology, and it has also been an important research topic in the field of image processing.

\section{Related Research}

Motion blur image restoration uses some priori knowledge of image degradation (such as the point spread function (PSF)) to recover the degraded image, which it is an urgent practical problems that needs to solve, but also it has important theoretical value. Compared to other forms of degraded images, motion blurred image acquisition process is caused by the relative motion, the complexity of the movement makes the point spread function is estimated to be much more difficult. Therefore, people have more extensive research and attention to the motion blur image restoration, and a variety of domestic and foreign research proposed restoration method has achieved some results. Based on the point spread function is intellectual, it can usually be divided into two categories: the first category is non-blind image restoration algorithm, which is exactly knowing the point spread function, then doing fuzzy image restoration . However, in reality, the point spread function is generally unknown, then the fuzzy image restoration called blind image restoration algorithm, that is the second-class restoration algorithm. The most critical and difficult step is blind image restoration algorithm, it is an estimate of the point spread function that causes the image produce motion blur, due to this characteristic that does not depend on the transfer function of the degradation system, so it has a wider application. The following describes the two types of research status related to image restoration methods at home and abroad.

\subsection{The Research of Non-blind Image Restoration Method}

Back in the mid-1960s, deconvolution (inverse filtering method) began to be widely used in the field of image restoration. Nathan used the two-dimensional inverse filtering method to recover the blurred image that Rover, Explorers and other extraterrestrial transmitter collected. At the same time, the image blur problem caused by atmospheric disturbances, Harris and Mcglamery adopt PSF analytical model and laboratory determined PSF deconvolution process, respectively. Since then, the inverse filtering method has become one of the most basic techniques of image restoration. However, although the inverse filtering method is relatively simple, but it did not consider interference, so the effect of recovery is poor when it has noise.

In 1967, Helstrom use minimum mean square error estimation, he put forward that using the Wiener filter for image restoration. The calculation is very small, and it has excellent resistance to noise. Later, the Slepian used the Wiener filtering to process random PSF condition [6]. With the Wiener filter in image restoration application, Habibi further improved Wiener filter, and improved the calculation speed. In short, in the case of obtaining the SNR of the degraded image, the restoration results of Wiener filter were quite good, but when the peak SNR is small, the effect of degraded image restoration is not very good. The above-mentioned non-blind image restoration method is the most basic, as the numerous studies, later there are a number of other ways, including five categories: statistical methods, maximum entropy method, regularization method and Kalman filtering methods etc.

(1) Statistical methods. It uses maximizing the probability to do image restoration in Bayesian framework conditions. Statistical methods include two types: the first one is the maximum likelihood method, which is designed to allow the resulting image that restored maximum possibly make the fuzzy image known can appear. And the method that using the known blurred image get the maximum possible 
original image, we called it that the maximum posteriori probability method, i.e., the second statistical method. 1970s, Richardson and Lucy raised that in the context of image pixels Poisson statistical distribution, using the maximum likelihood algorithm to image restoration, it is called Richardson-Lucy(RL) algorithm, and it is widely used in the image restoration at present. Lu and other scholars made improvements on the RL algorithm to reduce the ringing effect. Subsequently, a number of scholars have proposed priori probability that based on Markov field, generalized Gaussian models, and recovery methods of conditional probabilities that based on Gaussian models. However, these methods have much limit, and demand is high, if you want to get better results for image restoration, it requires that the prior probability model or conditional probability model is completely consistent with the original image.

(2) Maximum entropy method. It refers to all solutions in image restoration problem, there is always a solution that it's entropy is maximum, then this solution as the optimal solution, that is, after restoring the image to meet imaging conditions, the entropy is maximum. The maximum entropy is the restoration method that the problem of image restoration is to maximum entropy constrained normalized. In 1972, Frieden first made the maximum entropy method apply to image restoration, and proposed the maximum entropy algorithm that combined the object and noise restoration. Subsequently, Frieden and other scholars also proposed to use maximum entropy restoration problem transformed into the improvement of the deconvolution restoration [7], and get a maximum entropy fast algorithm that based on closed form solution. But Matthew and other scholars used first-order Taylor expansion instead of the original entropy expression, and also presents a maximum entropy fast algorithm. Li and other scholars proposed restoration method that using neural networks for solving maximum entropy. The maximum entropy method can effectively attenuate the noise and restore the image details, and it does not need too much assume the image priori knowledge. But the solution of numerical is more difficult, the calculation is very large, and very time consuming, so it is not suitable in some cases.

(3) Regularization method. It is based on variational, and established on the objective function which to solve the problem of blurred image [8], then added to appropriate a priori constraints, finally, using the most processing optimization obtained desired solution. Tikhonov first proposed to use regularization method deal with morbid inverse problems, then regularization method has become a typical and effective image restoration methods. Belge and other scholars maintain adaptive edge wavelet domain regularization method for image deblurring. Bioucas and other scholars have proposed a two-step iterative algorithm for obtaining the contraction threshold extremes image restoration model. There are many image deblurring algorithms based on regularization method, and regularization parameters largely affect the image restoration results. If regularization parameter value is too large, the image restoration result is smooth, making a lot of image detail is lost; the value is too small, image restoration results will contain high oscillation portion so that the restored image has a massive distortion.

Although the above restoration methods in various situations have achieved some results, but because of ill-posed problem of the transfer function [9], and the deviation between the actual value and the point spread function will affect the quality of the restored image, if the deviation is relatively large, the quality of the restored image is often unsatisfactory. Therefore, a lot of researches using some priori knowledge to solve this problem. 


\subsection{The Research of Blind Image Restoration Method}

When point extended function is unknown, the method make it restore the original image or the key information from the degraded image. Many practical applications are blind image restoration problems, it must extract degraded information from the degraded image was used to estimate the point spread function, that needs to identify accurately and efficiently two important parameters of the point spread function fuzzy scale and fuzzy angle. But compared with the non-blind image restoration method, the sick of blind image restoration method is more serious, because the point spread function is unknown, so the blind image restoration method is one of the hotspots and difficulties of current research in the field of image restoration.

The existing researches have put forward multiple blur identification method, according to the difference of main research methods used in blind image restoration, they can be divided into: the spatial domain method, frequency domain method, Bias estimation method and neural network method and so on.

(1) Spatial domain method. The image restoration method based on sharp edge in the width changes before and after the fuzzy edge. The original image edge width is small, but the degraded image edge width significantly increased, according to the variation characteristics, Lin and other scholars provide a method of detecting edge spread width, that solving the gradient change of each along the scanning line of object movement direction, then identifying the diffusion of the beginning and the end, finally, we can get the edge spread width, that is the approximate fuzzy scale. Bae used the multiscale edge detection and the fit of the model to estimate the blur parameters, to a certain extent, it improved the estimation accuracy of the fuzzy parameters.

(2) Frequency domain method. Due to Fourier transform map of fuzzy image have light and dark stripes, the method use this feature to analysis and estimate the fuzzy parameters. Cannon and other scholars have proposed that the spectrum of uniform linear motion blurred image exist regular zero value stripes, thus fuzzy scale is the central strip width of spectrum, fuzzy angle with zero value stripe is perpendicular to each other. This method applies only to blurred image with uniform linear motion, and noise immunity is poor. Since then, the researchers proposed a series of improved methods for the detection of spectral. Sakano and other scholars have proposed an improved method based on the Hough transform, they used dark stripes near gradient vector angular of blurred image spectrum to estimate fuzzy parameters [10], it improved the noise immunity. Lokhande and other scholars detected the dark stripes' direction of blurred images spectrum by Hough transform to estimate the blur angle. Moghaddam and other scholars detected the dark stripes' direction of blurred images spectrum by Randon transform to estimate the blur angle, and using fuzzy sets to estimate the scale. Tanka and other scholars have proposed to detect regular light and dark stripes that through the correlation between the spectrum of minimizing blurred image and the probe function.

(3) Bayesian estimation method. It is based on the observation data, using the sample information make the prior probability distribution of parameters change into posterior probability distribution of parameters. In 1763, Bayes first proposed the Bayesian theorem, the theorem through the observed values of binomial distribution to achieve probabilistic inference of parameters. With the improvement and development of Bayesian theory system, many researchers have proposed the blind source separation based on variational Bayesian theory, and then integrated priori knowledge of the original image pixel gray values, they achieved the blind image restoration of fuzzy image. Fergus and other scholars improved Miskin algorithm, the priori knowledge of original pixel gray values took the place of the priori knowledge of image gradient, using the overall learning parameter estimation 
method for image restoration, and achieved good results, but slowly, and the results of restoration is not particularly stable when point spread function is large. Shan and other scholars have proposed an improved maximum posterior probability estimation algorithm, it effectively solved the over-fitting of data, and inhibited the ripple, but it's convergence is slow, and setting of parameters will also affect the results.

(4) Neural network method. It uses a large number of essential nonlinear elements, which are organized in the form of a network, it likes the internet of neurons in the brain. In 1943, McCulloch and other scholars proposed neuron model, which uses binary threshold devices and 0-1 and 1-0 the random algorithm of neurological status mutation as the basis of the nervous system modeling. Through energy function, Zhou and other scholars connected the image restoration problems with the Hopfield neural network, so the image restoration problem is transformed into an optimization problem that the neural network can calculate. However, this method has vast network model, and it's convergence is very slow. Since then researchers proposed a series of improved blind image restoration method that based on neural network [11]. Moghaddam and other scholars proposed to estimate fuzzy parameters that using Randon transform and BP feedforward neural networks, especially for noisy images blurred scale identification based on Weierstrass approximation theorem and Steifel reference to design appropriate neural networks. Dash and other scholars proposed a novel fuzzy parameter estimation method, they used Gabor filters to estimate the blur angle, and designed a simple neural network to estimate the fuzzy scales.

(5) Other blind image restoration methods. In addition to the above methods, there are a lot of blind image restoration algorithms, such as image restoration method based on partial differential equations, image restoration method based on wavelet, image restoration method based on total variation and so on. With the rapid development of image restoration technology, some new ideas and algorithms are used to blind image restoration, this is not enumerate.

\section{The Proposed Scheme}

\subsection{Wavelet Transform}

Wavelet transform is a complex mathematical transform, it is widely used in science technology and engineering field. Wavelet transform can analyze the localization of the original signal in time and frequency, and it can show the local features of signal in time and frequency domain, so it easier to achieve the processing and analysis of signal. It has the characteristics of multi-resolution analysis.

Wavelet is the wave of the small region, it is a special waveform that has limited length (compactly supported), the mean is zero and fast decay. Wavelet function meaning: set $\psi(t)$ represents a square integrable function, $\psi(t) \subset L^{2}(R)$, if its Fourier transform $\psi(w)$ meets the following conditions:

$$
C_{\psi}=\int_{R} \frac{|\hat{\psi}(x)|^{2}}{|W|} d w<\infty
$$

$\psi(t)$ called basic wavelet or mother wavelet . Equation (1) is called wavelet function tolerable conditions. If translation and scaling of the basic wavelet function $\psi(t)$, we can get the wavelet base $\psi_{a, b}(t)$ :

$$
\psi_{a, b}(t)=\frac{1}{\sqrt{|a|}} \psi\left(\frac{t-b}{a}\right) \quad a, b \in R, \mathrm{a} \neq 0
$$


Where $a$ is scale factor; $b$ for displacement factor. Below is a list of several common wavelet base, as shown in Table 1 .

Table 1. Wavelet Base Function

\begin{tabular}{|c|c|}
\hline Serial number & Wavelet base name \\
\hline 1 & Haar \\
\hline 2 & Daubechies(dbN) \\
\hline 3 & Morlet \\
\hline 4 & Meyer \\
\hline 5 & Symlet \\
\hline 6 & Coiflet \\
\hline 7 & Biorthogonal \\
\hline
\end{tabular}

\subsection{Cepstrum}

The cepstrum of blurred image $\mathrm{g}(\mathrm{x}, \mathrm{y})$ :

$$
C_{g}(p, q)=F^{-1}[\lg |G(\mu, v)|]
$$

Where $G(\mu, v)$ represents Fourier transform of blurred image $\mathrm{g}(\mathrm{x}, \mathrm{y}), F^{-1}$ is the inverse transformation of Fourier transform. From equation ( 3 ) we can see, for the Fourier transform modulus value of an image take logarithm, and then doing the inverse transform of Fourier transform, we can get cepstrum of blurred image. The basic flow of generating the cepstrum of an image shown in Figure 1.

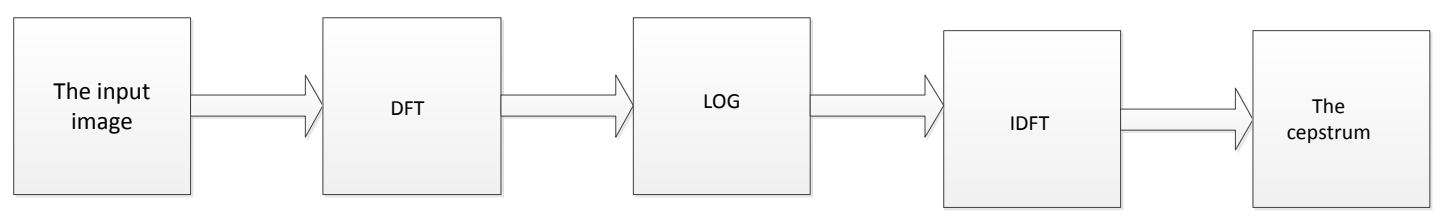

Figure 1. Flow Chart of Cepstrum Generation

\subsection{The Design of Point Expansion Function Identification Algorithm for Parameters based on 2D-DWT and Cepstrum}

Wavelet transform, wavelet of various types can construct different filters to obtain the approximate part of motion blurred image, and it is applied to the subsequent identification of the point spread function. Therefore, in practical applications, the two key factors of the 2D-DWT transform of the motion blurred image is the choice of wavelet base and the determination of decomposition level. The different wavelet basis, and different decomposition level will directly affect the identification precision of final point spread function.

\section{(1) The Selection of 2D-DWT wavelet}

Various wavelet has different time-frequency characteristics, from Tab 1, there are a lot of wavelet type, we select different wavelet bases to analyze the same problems, it will produce different results. Therefore, there is still no good wavelet base selection guidance theory, in general, we use common wavelet to do experimental comparative and analysis, and select the best wavelet basis for the 2D-DWT transform of motion blurred image.

This paper selects Cameraman images as the experimental object, different fuzzy scale $(10 \leq L \leq 60)$, uniform linear motion of different fuzzy angle $(0 \leq \theta \leq 180)$, and 
then doing a layer of 2D-DWT transform of the 48 pieces motion blurred image, at last using different wavelet basis to obtain approximate part apply to identify parameters of point spread function. Tab 2 gives the different wavelet basis, the maximum differential error of PSF parameters of 48 pieces motion blurred image. Among them, the number of gross error represents using the wavelet to do parameter identification of PSF, and we get fuzzy scale and fuzzy angle of big deviation from differential data.

Table 2. Accuracy of Identification with Different Wavelet Basis Functions

\begin{tabular}{|c|c|c|}
\hline Wavelet base & $\begin{array}{c}\text { The maximum error of the } \\
\text { differential }\end{array}$ & Gross error number \\
\hline $\mathrm{db} 1$ & $1 / 2$ & 0 \\
\hline $\mathrm{db} 2$ & $1 / 2$ & 4 \\
\hline $\mathrm{db} 4$ & $4 / 7$ & 9 \\
\hline sym2 & $2 / 2$ & 3 \\
\hline coif 1 & $2 / 5$ & 8 \\
\hline bior1.1 & $1 / 2$ & 0 \\
\hline
\end{tabular}

From the results in Table 2, we made 48 pieces motion blurred images that have different fuzzy scale, different fuzzy angle do parameter identification of PSF, identify precision of $\mathrm{db} 1, \mathrm{db} 2$ and bior 1.1 was the highest, The maximum identification error of fuzzy scale and fuzzy angle were 1 pixels, $2^{0}$, respectively, but $\mathrm{db} 2$ had 4 groups data appeared gross error, so the identification precision and effectiveness of $\mathrm{db} 1$ and bior 1.1 were the highest. the identification precision of $\mathrm{db} 4$ was the lowest, this is because the blurred image after using db4 wavelet 2D-DWT, approximate part had abundant information, but it also had much redundant information, it not only made the operation speed is slow, but also made the identification precision is reduced. At the same time, the wavelet db6 and bior3.7 experiments were carried out, the identification precision is particularly low, and the operation speed is not ideal.

\section{(2) The Determination of 2D-DWT Decomposition Level}

Another key factor that affects the 2D-DWT apply to identify parameters of the motion fuzzy image point spread function when we determine the wavelet decomposition level. We can obtain from Fig.3.3 and 3.4, through a layer of 2D-DWT, we can reduce the dimensionality, the approximate part focused on the content information of the image. Although using excessive decomposition level can be further dimension reduction, but it will lead to useful information for each part of the decomposition contains too little, especially for the fuzzy part of the follow-up feature information approximate point spread function parameter identification, and the much decomposition level will increase computing time of identification procedures .In order to determine the optimal decomposition level, this section is still selected above 48 pieces motion blurred images as experimental subjects, conducted experiments in different decomposition levels, respectively, and compared with the point spread function parameter identification results, the results are shown in Table 2. 
Table 3. Precision of Identification with Different Decomposition Level

\begin{tabular}{|c|c|c|}
\hline Decomposition layers & $\begin{array}{c}\text { The maximum error of the } \\
\text { differential }\end{array}$ & Gross error number \\
\hline Don't break down & $2 / 5$ & 2 \\
\hline 1 & $1 / 2$ & 0 \\
\hline 2 & $6 / 7$ & 8 \\
\hline
\end{tabular}

From the results in Table 3 , in PSF parameter identification of the blur 48 different scales, different angles of motion blurred image, identification precision of a layer 2D-DWT is highest. Do not use 2D-DWT and the two layer 2D-DWT will have gross error, identification accuracy and stability are decreased. Therefore, using a layer of 2D-DWT, both noise, reducing dimension and saving time.

Through a layer 2D-DWT, the vast majority of useful information of motion blurred image locates in approximate section, details shares the minority, and most of it is the image noise, so we can give up details, and just take the approximate part to do the point spread further function parameter identification, thus it not only can improve identification precision, but also improve the effectiveness.

\subsection{Algorithm Implementation}

Based on the above discussion, based on 2D-DWT and cepstrum point spread function parameter identification algorithm, follow these steps :

(1) Motion blurred image $\mathrm{g}(\mathrm{x}, \mathrm{y})$ do a layer 2D-DWT transform, we get the approximate part $\mathrm{A} 1$ of motion blurred image $\mathrm{g}(\mathrm{x}, \mathrm{y})$;

(2) By calculating, we get the approximate part A1'cepstrum $C_{g}(p, q)$ of the motion blurred image, and making $\mathrm{p}=0, \mathrm{q}=0$ move to the center position ;

(3) Doing Canny edge detection with cepstrum maps, we get cepstrum maps' edge detection image e $(x, y)$;

(4) Using mathematical morphology to do expansion operations for edge detection image e (x,y), we get 2 negative peaks $H_{\max 1}\left(p_{1}, q_{1}\right)$ and $H_{\max 2}\left(p_{2}, q_{2}\right)$;

(5) According to the distance between $H_{\max 1}$ and $H_{\max 2}$, which is 2 times of the fuzzy scale L, then:

$$
L=\frac{1}{2} \sqrt{\left(p_{1}-p_{2}\right)^{2}+\left(q_{1}-q_{2}\right)^{2}}
$$

(6) According to the angle between $H_{\max 1}$ and $H_{\max 2}$, which is fuzzy angle $\theta$,then:

$$
\theta=\arctan \frac{p_{1}-p_{2}}{q_{1}-q_{2}} \times \frac{180}{\pi}
$$

\section{Experimental Results and Analysis}

\subsection{The Results of Noiseless Motion Blurred Image Restoration}

In the absence of noise situation, this paper selects the image of the standard database (Cameraman), doing uniform linear motion with different fuzzy scale $(10 \leq L \leq 60)$, different fuzzy angle $(0 \leq \theta \leq 180)$, Then using the proposed point spread function parameter identification method for the parameter identification of PSF against multiple computer simulation fuzzy images, finally, we obtained fuzzy parameter which used identification, using the classical image restoration method 
for restoration. Using inverse filtering, Wiener filter for the restoration, respectively, the rehabilitation results are as follows:

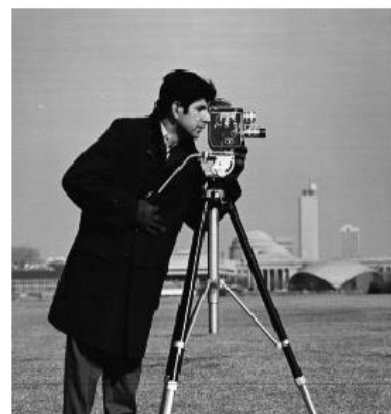

a) original image

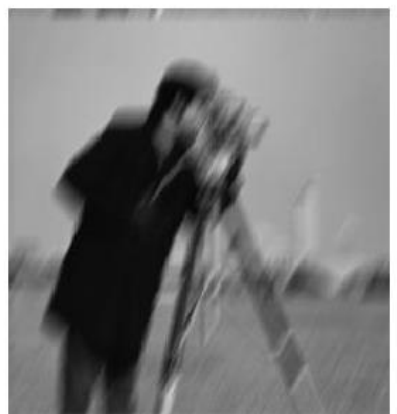

b) moving blurred

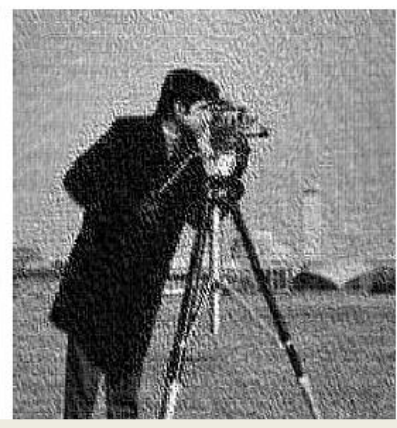

c) Inverse filtering

Figure 2. Cameraman Image Restoration Inverse Filtering Results

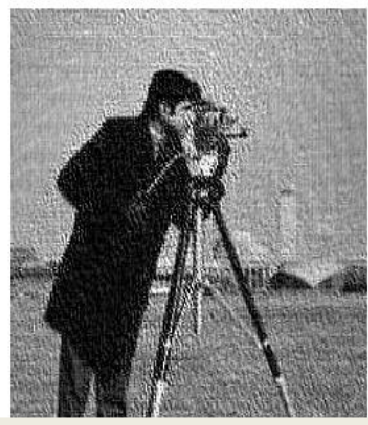

a) $\mathrm{k}=0.0001$

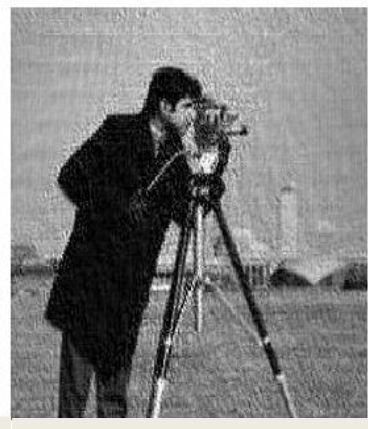

b) $\mathrm{k}=0.001$

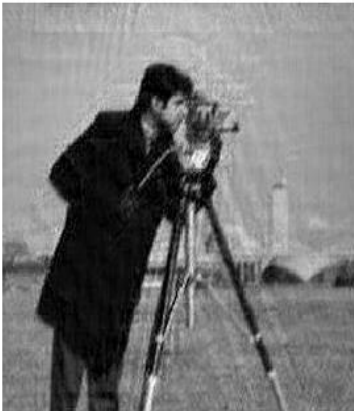

a) $\mathrm{k}=0.005$

Figure 3. Cameraman Image Restoration Wiener Filter Results

Inverse filtering can be drawn on the motion blurred image restoration exist ringing effects, but the restored image is clear and sharp, the overall effect is good. Wiener filter can obtain the best recovery results by adjusting the Wiener filtering parameters $\mathrm{k}$. The greater parameter $\mathrm{k}$, the restoration image is more unclear, and the outline is more unclear. And the parameter $\mathrm{k}$ is too large, it will lead to PSNR declined, the noise will be amplified.

\subsection{Noisy Motion Blurred Image Restoration Results}

For Cameraman $\left(L^{\prime}=21, \theta^{\prime}=60\right)$, adding the Gaussian noise that it's mean is zero, and noise variance is 0.002 , it has been used the point spread function of parameter identification method in this paper. Using Inverse filter, Wiener filter for restoration, respectively, restoration results are as follows: 


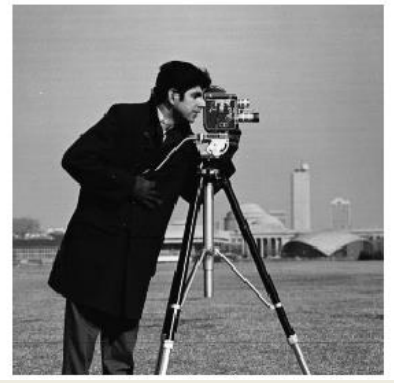

a) original image

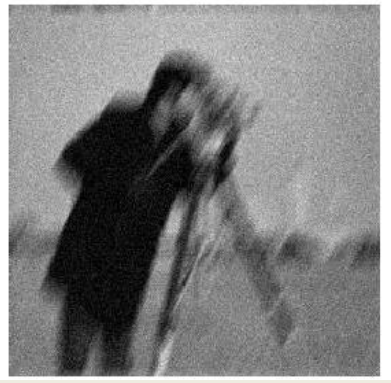

b) moving blurred image with noise

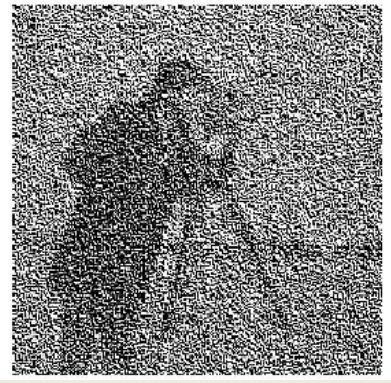

c) Inverse filtering restoration

Figure 4. Cameraman Image Restoration Inverse Filtering Results with Gaussian Noise $(v=0.002)$

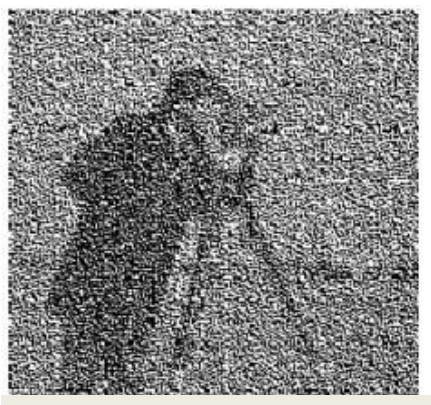

a ) $(\mathrm{k}=0.0001)$

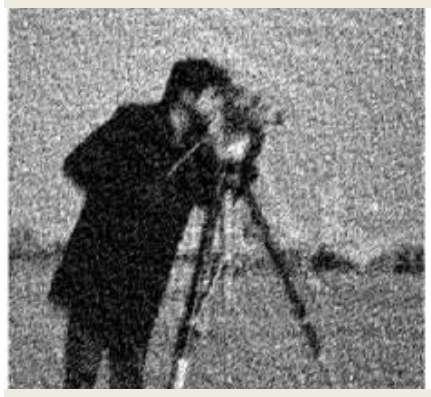

d) $(\mathrm{k}=0.01)$

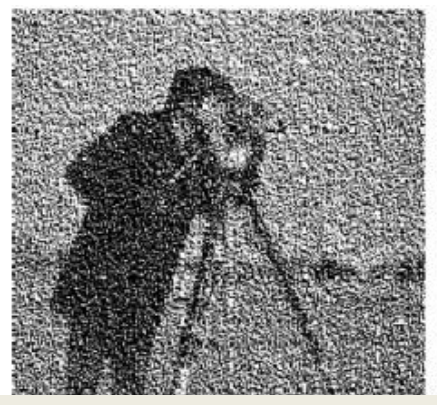

b) $\quad(\mathrm{k}=0.001)$

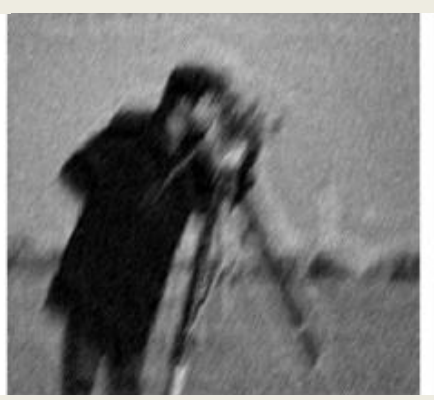

e) $(\mathrm{k}=0.1)$

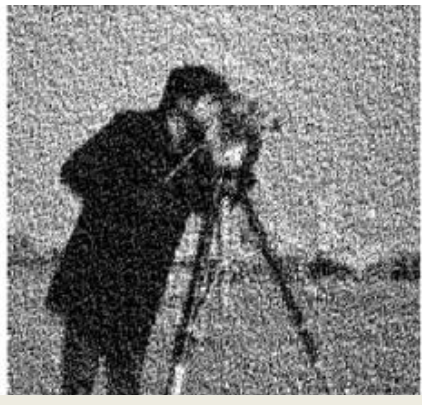

c) $(\mathrm{k}=0.005)$

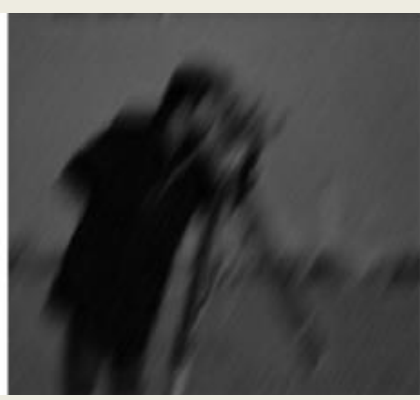

f) $(\mathrm{k}=1)$

\section{Figure 5. Cameraman Image Restoration Wiener Filter Results Containing} Gaussian Noise ( $v=0.002)$

Inverse filtering can be drawn on the noisy image restoration motion blurred effect is poor, it unable to suppress noise, the clarity of restoration image is poor. Wiener filter can obtained better restoration effect by adjusting the parameters $\mathrm{k}$, then it can effectively suppress noise. When the parameter $\mathrm{k}$ is different, the difference of restoration effect is relatively large, $\mathrm{k}$ is too large or too small, the image sharpness will be reduced, the profile is not clear. So we can know, when the parameter $\mathrm{k}=0.01$, the recovery is best, and after restoring image ,the clarity and contours are ideal.

\section{Conclusions}

This paper discusses the two kinds of classical image restoration methods: inverse filtering restoration, Wiener filtering restoration, we give the principle and formula derivation of the algorithm. Then using proposed identification method in this paper 
identified obtained PSF parameters, using inverse filtering, Wiener filtering computer simulation motion blurred image without noise and noisy conducted a simulation experiment, the results are given in restoration results maps. At the same time, selecting PSNR, MSE, GMG, LS four kinds of image quality assessment methods evaluated the effect of restoration, we gave the restored image quality evaluation results. Finally, we made the actual shooting motion blurred images do PSF parameter identification, RL and filter restoration results further demonstrate the effectiveness of the proposed point spread function parameter identification methods.

\section{References}

[1] Y. Yitzhaky, R. Milberg and S. Yohaev, "Comparison of direct blind deconvolution methods for motion-blurred images [J]", Applied optics, vol. 38, no. 20, (1999), pp. 4325-4332.

[2] D. Calvetti, B. Lewis and L. Reichel, "GMRES-type methods for inconsistent systems [J]", Linear Algebra and its Applications, vol. 316, no. 1, (2000), pp. 157-169.

[3] M. Hanke, J G. Nagy and C. Vogel, "Quasi-Newton approach to nonnegative image restorations [J]", Linear Algebra and its applications, vol. 316, no. 1, (2000), pp. 223-236.

[4] M. Rojas and T. Steihaug, "An interior-point trust-region-based method for large-scale non-negative regularization [J]", Inverse Problems, vol. 18, no. 5, (2002), p. 1291.

[5] A. Dax, "The convergence of linear stationary iterative processes for solving singular unstructured systems of linear equations [J]", SIAM review, vol. 32, no. 4, (1990), pp. 611-635.

[6] Y Q. Lin, "Implicitly restarted global FOM and GMRES for nonsymmetric matrix equations and Sylvester equations [J]", Applied mathematics and computation, vol. 167, no. 2, (2005), pp. 1004-1025.

[7] M. Bellalij, K. Jbilou and H. Sadok, "New convergence results on the global GMRES method for diagonalizable matrices [J]", Journal of Computational and Applied Mathematics, vol. 219, no. 2, (2008), pp. 350-358.

[8] A. Bouhamidi, K. Jbilou and S. Tikhonov. "Regularization methods in image restoration [J]", Journal of computational and applied mathematics, vol. 206, no. 1, (2007), pp. 86-98.

[9] M. Christiansen and M. Hanke, "Deblurring methods using antireflective boundary conditions [J]", SIAM Journal on Scientific Computing, vol. 30, no. 2, (2008), pp. 855-872.

[10] M. Muneyasu, N. Nishi and T. Hinamoto, "A new adaptive center weighted median filter using counter propagation networks [J]", Journal of the Franklin Institute, vol. 337, no. 5, (2000), pp. 631-639.

[11] J G. Nagy, K. Palmer and L. Perrone, "Iterative methods for image deblurring: a Matlab object-oriented approach [J]. Numerical Algorithms, vol. 36, no. 1, (2004), pp. 73-93.

\section{Authors}

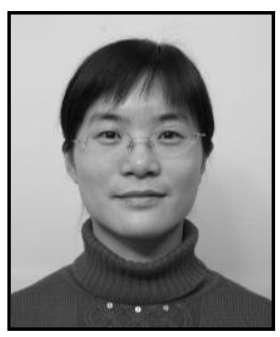

Qiong Li, she received her B.Eng degree in Electrical Engineering from Guangxi Normal University and M.Eng degree in Electrical Engineering from Guilin University of Electronic Technology, china. Her current research interest is image processing.

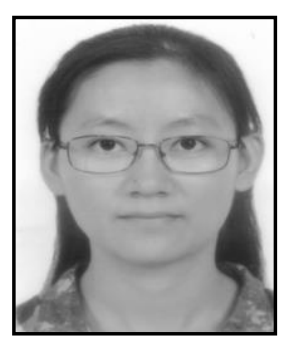

Junhui Rao, she received her B.Sc degree in Information science and technology from North China Electric Power University and M.Eng degree in Electronic information science and technology from Sun Yat-sen University, China, in 2004 and 2006 respectively. She is currently researching on image processing and information hiding. 


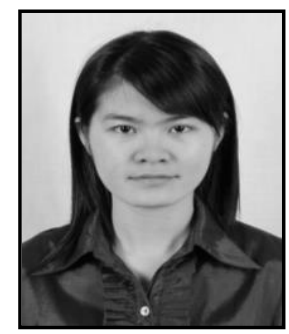

Xiaofeng Yang, she received her B.Eng degree in Electrical Engineering from Tongji University and M.S degree in Electrical Engineering from Michigan Technological University. She is currently a lecturer with Yulin Normal University. Her researc $\mathrm{h}$ interests include Array Signal Processing and UWB Positioning. 
International Journal of Signal Processing, Image Processing and Pattern Recognition Vol.8, No.9 (2015) 